\title{
Habilidades Sociais em Alcoolistas: Um Estudo Comparativo ${ }^{1}$
}

\author{
Flaviane Bevilaqua Felicissimo ${ }^{2}$ \\ Juliana Aparecida dos Santos \\ Laiz de Oliveira Fontoura \\ Telmo Mota Ronzani \\ Universidade Federal de Juiz de Fora
}

\begin{abstract}
RESUMO - O presente estudo objetivou comparar o repertório de habilidades sociais (HS) de dependentes e não dependentes de álcool a fim de verificar se um menor repertório de HS constitui uma característica dessa população. Foram entrevistados 123 alcoolistas e 114 usuários de serviços de saúde com baixo ou nenhum consumo de álcool, utilizando questionário sociodemográfico, Mini International Neuropsychiatric Interview e o Inventário de Habilidades Sociais. Os dados foram analisados quantitativamente e indicaram uma diferença significativa no repertório de HS, especificamente no fator de autocontrole da agressividade, indicando um pior desempenho dessa habilidade em alcoolistas. Os resultados sugerem que a avaliação dessa característica deva ser considerada no tratamento do alcoolismo e associada ao plano terapêutico.
\end{abstract}

Palavras-Chave: alcoolismo, habilidades sociais, Inventário de Habilidades Sociais

\section{Social Skills of Alcoholics: A Comparative Study}

\begin{abstract}
The present study aimed to compare the repertoire of social skills of alcoholics and non-alcoholics in order to verify if a minor repertoire of social skills constitute a feature of this population. Interviews were carried out with 123 alcoholics and 114 users of health service patients with low or no consumption of alcohol. It were used a socio-demographic questionnaire, the Mini International Neuropsychiatric Interview and the Social Skill Inventory. The data were analyzed quantitatively and the results indicated a significant difference in repertoire of social skills, specifically with respect to the factor of self-control of aggressiveness, on which was demonstrated the worst performance. The results suggest that the evaluation of this feature should be considered in the treatment of alcoholism and associated with a therapeutic plan.
\end{abstract}

Keywords: alcoholism, social skills, Social Skill Inventory

O álcool está entre as poucas substâncias psicotrópicas que têm seu consumo admitido e, muitas vezes, incentivado pelas sociedades ocidentais (Scali \& Ronzani, 2007). No entanto, essa substância possui uma função ambígua, em que, por um lado, promove a socialização, alegra e comemora e, por outro, também pode originar um grave transtorno de saúde pública mundial (Gigliottia \& Bessa, 2004).

O consumo em grande quantidade e frequente de álcool afeta praticamente todos os órgãos do corpo humano em curto e longo prazo (World Health Organization [WHO], 2014), porém, paradoxalmente, muitas pessoas esperam por consequências diretas advindas desse consumo para considerá-lo prejudicial à saúde. Sabe-se que as consequências negativas de beber, como os sintomas da abstinência, depressão e ansiedade, podem aparecer um longo tempo após o consumo pesado e habitual, mas, mesmo assim, não suplantam o reforço positivo imediato causado pelos efeitos do álcool (Kadden \& Cooney, 2005). Ainda, diversas consequências indiretas podem ser atribuíveis a esse

1 Agradecimentos: À Coordenação de Aperfeiçoamento de Pessoal de Nível Superior (CAPES) e ao Conselho Nacional de Desenvolvimento Científico, Tecnológico $(\mathrm{CNPq})$ e Fundação de Apoio à Pesquisa de Minas Gerais (FAPEMIG); Ao Psicólogo Leonardo Fernandes Martins pela contribuição nas análises estatísticas

2 Endereço para correspondência: Rua das Turquesas, $n^{\circ} 205$, Marilândia, Juiz de Fora, MG, Brasil. CEP: 36.039-310. E-mail: flavianejf@ hotmail.com consumo exagerado, como é o caso de acidentes de trânsito, violência, comportamentos de risco, entre outros (Duarte, Stempliuk, \& Barroso, 2009).

Além das consequências diretas e indiretas provocadas aos indivíduos que fazem consumo pesado de álcool, existem consequências indiretas também para aqueles com quem estes convivem. Como exemplo, pode-se citar os passageiros envolvidos nos acidentes de trânsito, membros da família afetados pelo descumprimento de seu papel social e incidentes de violência doméstica (WHO, 2014).

Cunha, Peuker e Bizarro (2012) destacaram, entre os prejuízos decorrentes e agravantes do abuso de álcool, os comprometimentos nos relacionamentos interpessoais. Assim, os autores explicaram que prejuízos no repertório de habilidades sociais podem constituir-se em um importante fator de risco para o abuso de álcool, uma vez que indivíduos com tais déficits podem fazer uso dessa substância como estratégia para enfrentar interações sociais e, assim, diminuir a tensão gerada por essas situações (Cunha, Carvalho, Kolling, Silva, \& Kristensen, 2007).

Frequentemente, os consumidores de álcool apresentam dificuldades em estabelecer relações sociais de acordo com o que se espera deles, gerando respostas sociais aprendidas de forma parcial e inadequada (Caballo, 2010). Um ciclo vicioso se forma nesse contexto, em que o indivíduo que bebe com frequência seleciona amigos entre as pessoas que bebem muito, diminuindo a probabilidade de aprender um 
comportamento social adequado sem recorrer ao álcool, favorecendo problemas que interferem no funcionamento social e de trabalho do sujeito (Caballo, 2010).

Algumas contribuições atuais à área dos transtornos associados ao uso de substâncias estão sendo desenvolvidas através de pesquisas que buscam identificar se a existência de déficits no repertório de habilidades sociais constitui-se em um importante fator de risco para o desenvolvimento de um padrão abusivo e/ou de dependência do álcool (Aliane, Lourenço, \& Ronzani, 2006). No entanto, poucos são os estudos desenvolvidos no contexto brasileiro que buscam verificar essa relação empiricamente.

Em uma revisão realizada por Felicissimo, Casela e Ronzani (2013), foram encontrados apenas dois estudos brasileiros que tinham como objetivo caracterizar o repertório de habilidades sociais em dependentes de álcool. Os dois estudos em questão (Aliane et al., 2006; Cunha et al., 2007) utilizaram o mesmo instrumento para caracterizar o desempenho social dos entrevistados: o Inventário de Habilidades Sociais- IHS (Del Prette \& Del Prette, 2001). No entanto, encontraram resultados distintos.

O estudo de Aliane et al. (2006) comparou o repertório de habilidades sociais de dependentes de álcool ao de indivíduos não dependentes, mas que tinham o mesmo perfil sociodemográfico. Não se encontraram diferenças estatisticamente significativas entre os dois grupos. Foram encontradas diferenças apenas entre os gêneros, em que os homens apresentaram uma média maior do que as mulheres no fator "conversação e desenvoltura social" e "autocontrole da agressividade". Todavia, pode-se considerar este como um estudo inicial, pois apresentou uma importante limitação relacionada ao tamanho da amostra.

Por outro lado, o estudo de Cunha et al. (2007) investigou a existência de déficits em habilidades sociais em uma amostra de dependentes de álcool comparados aos dados normativos do IHS e encontrou uma maior evidência desses déficits nos fatores de "autoafirmação de sentimento positivo" e "conversação e desenvoltura social". Esses resultados indicaram que o álcool atua como uma estratégia de enfrentamento em situações ansiogênicas, visto que os participantes desse estudo concordavam que o álcool facilitava suas interações sociais, deixava-os mais confiantes e desinibidos, favorecendo suas relações interpessoais (Cunha et al., 2007). No entanto, esse estudo apresenta uma importante limitação: o grupo de alcoolistas foi comparado à amostra normativa do IHS, composta por uma população de jovens universitários com idade entre 17 e 25 anos. Esse perfil difere significativamente do perfil encontrado em amostras de dependentes de álcool, que, em sua maioria, é composto por indivíduos com baixa escolaridade, predominância do sexo masculino e na faixa etária correspondente à idade adulta (Monteiro et al., 2011; Laranjeira, Pinsky, Zaleski, \& Caetano, 2007; Costa et al., 2004). Esses dados indicam uma significativa limitação na comparação de indivíduos dependentes de álcool com a amostra normativa do IHS.

Sendo assim, percebe-se que os dados existentes apresentam importantes limitações, além de uma inconsistência nos resultados acerca da relação entre o repertório de habilidades sociais e a dependência do álcool, o que impulsionou o desenvolvimento do presente estudo, que tem como objetivo principal comparar o repertório de habilidades sociais entre dependentes e não dependentes de álcool contando com uma amostra superior aos estudos supracitados.

\section{Método}

\section{Participantes}

Participaram desta pesquisa 237 pacientes divididos em dois grupos distintos. O primeiro grupo foi constituído por 123 pacientes com diagnóstico de dependência de álcool, que estavam em tratamento em instituições especializadas em dependência de substâncias na cidade de Juiz de Fora MG e que preencheram os seguintes critérios de inclusão: (a) ter recebido o diagnóstico de dependência de álcool; (b) ser usuário de serviços de tratamento especializados em dependência; (c) ter dado entrada no serviço de tratamento no mês da coleta de dados para evitar vieses de tratamento e (d) ser maior de 18 anos. O segundo grupo foi composto por 114 usuários dos serviços de atenção primária à saúde no município de Juiz de Fora-MG, que contemplaram os seguintes critérios de elegibilidade: (a) não ter diagnóstico de dependência de álcool ou outras substâncias psicoativas (exceto tabaco); (b) ser maior de 18 anos; (c) estar em busca de atendimento em alguma unidade básica de saúde e (d) ter o mesmo perfil da amostra de dependentes de álcool quanto ao sexo, idade e escolaridade. Os grupos foram pareados em relação ao sexo, idade e escolaridade, tendo-se apresentados homogêneos quanto a essas variáveis, o que tornou mais confiável a comparação entre ambos.

\section{Instrumentos}

Foram utilizados três instrumentos neste estudo, a saber:

1. Questionário sóciodemografico: utilizado para a caracterização dos participantes da pesquisa quanto a idade, gênero, estado civil, status profissional, religião, renda familiar, escolaridade e anos de educação.

2. Mini International Neuropsychiatric Interview (MINI): utilizado como critério de elegibilidade para o estudo. Consiste em um questionário breve (15-30 minutos) com perguntas dicotômicas (SIM/ NÃO) compatíveis com os critérios diagnósticos do DSM-IV-R e da CID-10. O MINI apresenta qualidades psicométricas comparáveis às de outros questionários diagnósticos padronizados mais longos e complexos, permitindo a avaliação rápida dos principais transtornos do Eixo I do DSM-IV (Amorim, 2000).

3. Inventário de Habilidades Sociais (IHS): instrumento brasileiro cujo objetivo é caracterizar o repertório de habilidades sociais em situações pessoais cotidianas (Del Prette \& Del Prette, 2001). É composto por 38 itens respondidos em uma escala likert de 
cinco pontos, variando de "nunca ou raramente" a "sempre ou quase sempre", que descrevem uma série de situações sociais. Os itens são agrupados em cinco subescalas ou fatores: F1) Enfrentamento e Autoafirmação com Risco; F2) Autoafirmação na Expressão de Sentimento Positivo; F3) Conversação e Desenvoltura Social; F4) Autoexposição a desconhecidos e situações novas; e F5) Autocontrole da Agressividade. No presente estudo, o alfa de Cronbach para a amostra de dependentes de álcool e não dependentes foram, respectivamente, $\alpha=0,78$ e 0,72 .

\section{Procedimentos}

O projeto foi submetido ao Comitê de Ética em Pesquisa da Universidade Federal de Juiz de Fora e aprovado (Parecer $\mathrm{n}^{\circ}$ 057/2010). Todos os participantes assinaram o Termo de Consentimento Livre e Esclarecido, assegurando o anonimato e os preceitos éticos em pesquisa. Ambos os grupos foram abordados durante o momento de espera por atendimento nos serviços de saúde e a realização das entrevistas foi efetuada conforme a disponibilidade dos participantes. Os questionários foram aplicados em forma de entrevista estruturada, respeitando-se as instruções para aplicação dos instrumentos utilizados.

Tabela 1. Descrição da amostra de dependentes e não dependentes

\section{Análises dos dados}

Os dados coletados foram codificados por dois digitadores de forma independente em um banco de dados da versão 15.0 do software Statistical Package for Social Science SPSS $®$. As duas versões da digitação foram submetidas a um processo de comparação utilizando o software Epinfo 3.5 para detectar e corrigir eventuais erros de digitação. Após o banco consolidado, os dados foram submetidos a análises estatísticas exploratórias através de estatísticas descritivas, sendo elas: frequência absoluta (n), frequência relativa (\%), média (M) e desvio padrão (DP). Para as análises estatísticas inferenciais, no caso das variáveis numéricas, foi utilizado o Teste " $t$ " de Student, quando os pressupostos de normalidade e homogeneidade de variância foram preenchidos. Quando tais pressupostos não foram observados, utilizou-se o teste não-paramétrico de Mann-Whitney. Para as variáveis categóricas, foi utilizado o teste estatístico do Qui-Quadrado $\left(X^{2}\right)$. Ainda, para verificar se a distribuição dos dados amostrais diferia de uma distribuição normal, optou-se por utilizar o teste Kolmogorov-Smirnov. Por último, a fim de avaliar a consistência interna do IHS utilizou-se o alpha de Cronbach. Adotou-se, para todas as análises inferenciais, o intervalo de confiança de $95 \%(\mathrm{p}<0,05)$.

\begin{tabular}{|c|c|c|c|c|c|}
\hline & \multirow[b]{2}{*}{ Características } & \multirow{2}{*}{$\begin{array}{c}\begin{array}{c}\text { Dependentes } \\
(\mathbf{n}=\mathbf{1 2 3})\end{array} \\
\% \\
\end{array}$} & \multirow{2}{*}{$\begin{array}{c}\text { Não dependentes } \\
(\mathrm{n}=114)\end{array}$} & \multicolumn{2}{|c|}{$X^{2}$ Pearson } \\
\hline & & & & $X^{2}$ & $\mathrm{p}$ \\
\hline \multirow[t]{2}{*}{ Sexo } & Feminino & 8,9 & 8,8 & 0,00 & 0,97 \\
\hline & Masculino & 91,1 & 91,2 & & \\
\hline \multirow[t]{4}{*}{ Estado Civil } & Solteiro(a) & 33,3 & 24,6 & 21,29 & 0,00 \\
\hline & $\begin{array}{r}\text { Casado(a)/Vive com } \\
\text { companheiro(a) }\end{array}$ & 42,3 & 65,8 & & \\
\hline & Divorciado(a) & 23,6 & 7,0 & & \\
\hline & Viúvo(a) & 0 & 2,6 & & \\
\hline \multirow[t]{2}{*}{ Atualmente, está trabalhando? } & Sim & 30,1 & 54,4 & 13,65 & 0,001 \\
\hline & Não & 68,3 & 45,6 & & \\
\hline \multirow[t]{5}{*}{ Renda Familiar } & Até um sal. mínimo & 18,7 & 23,7 & 1,31 & 0,86 \\
\hline & 1 a 3 sal. mínimos & 53,7 & 56,1 & & \\
\hline & 4 a 6 sal. mínimos & 16,3 & 17,5 & & \\
\hline & 7 a 10 sal. mínimos & 1,6 & 1,8 & & \\
\hline & Mais de 10 sal. mínimos & 0 & ,9 & & \\
\hline \multirow[t]{7}{*}{ Escolaridade } & $1^{\circ}$ a $4^{\circ}$ Série Incompleto & 9,8 & 11,4 & 2,27 & 0,68 \\
\hline & $1^{\circ}$ a $4^{\circ}$ Série Completo & 15,4 & 18,4 & & \\
\hline & $5^{\circ}$ a $8^{\circ}$ Série Incompleto & 35,0 & 35,1 & & \\
\hline & $5^{\circ}$ a $8^{\circ}$ Série Completo & 13,8 & 8,8 & & \\
\hline & Ensino Médio Incompleto & 7,3 & 7,9 & & \\
\hline & Ensino Médio Completo & 13,8 & 14,9 & & \\
\hline & Superior Incompleto & 4,9 & 3,5 & & \\
\hline
\end{tabular}

Nota. Para algumas variáveis, o percentual está baseado em um n menor devido ao missing dos dados 


\section{Resultados}

Conforme é apresentado na Tabela 1, não foram encontradas diferenças significativas quanto ao sexo, renda e escolaridade entre os grupos. Ainda, a amostra foi predominantemente do sexo masculino, com idade média para a amostra de não dependentes de 44,2 anos $(\mathrm{DP}=11,7)$ e para a amostra de dependentes de 43,2 anos (DP=9). O número de vezes que o entrevistado buscou algum tratamento para problemas relacionados ao uso de álcool variou de "nenhuma procura" a 25 tentativas, com média de 3,1 tentativas $(\mathrm{DP}=3,98)$. Entre as tentativas realizadas, a maioria $(60,9 \%)$ relatou já ter permanecido internado em algum momento. O tempo mínimo de internação variou de um dia a três meses, e o tempo máximo foram dois anos de internação. Entre os serviços procurados com maior frequência, encontram-se o CAPS-ad (51,2\%), Hospital psiquiátrico (49,6\%), Hospital de Pronto Socorro $(43,1 \%)$ e Unidades Básicas de Saúde (27,6\%).

Com relação ao repertório de habilidades sociais, só foram encontradas diferenças significativas no fator representante da habilidade de autocontrole da agressividade, indicando um pior desempenho dessa habilidade em indivíduos alcoolistas. Para o escore total do IHS e os demais escores fatoriais, não houve diferenças significativas, conforme pode ser visualizado na Tabela 2.

Tabela 2. Descrição do repertório de Habilidades Sociais de dependentes e não dependentes de álcool

\begin{tabular}{|c|c|c|c|}
\hline & & Dependentes & $\begin{array}{c}\text { Não } \\
\text { dependentes }\end{array}$ \\
\hline \multirow[t]{4}{*}{ IHS-Total } & Média & 94,93 & 96,14 \\
\hline & DP & 18,98 & 17,06 \\
\hline & Valor Mín. & 32,44 & 51,0 \\
\hline & Valor Máx. & 147,0 & 132,0 \\
\hline \multirow{4}{*}{$\begin{array}{l}\text { F1 - Enfrentamento e } \\
\text { Autoafirmação com } \\
\text { Risco }\end{array}$} & Média & 2,29 & 2,24 \\
\hline & DP & 0,73 & 0,72 \\
\hline & Valor Mín. & 0,36 & 0,36 \\
\hline & Valor Máx. & 4,0 & 3,64 \\
\hline \multirow{4}{*}{$\begin{array}{l}\text { F2- Autoafirmação } \\
\text { na Expressão de } \\
\text { Sentimento Positivo }\end{array}$} & Média & 2,30 & 3,08 \\
\hline & DP & 0,70 & 0,71 \\
\hline & Valor Mín. & 0,57 & 0,71 \\
\hline & Valor Máx. & 4,0 & 4,0 \\
\hline \multirow{4}{*}{$\begin{array}{l}\text { F3-Conversação e } \\
\text { Desenvoltura Social }\end{array}$} & Média & 2,48 & 2,62 \\
\hline & DP & 0,89 & 0,91 \\
\hline & Valor Mín. & 0 & 0,43 \\
\hline & Valor Máx. & 4,0 & 4,0 \\
\hline \multirow{4}{*}{$\begin{array}{l}\text { F4- Autoexposição } \\
\text { a desconhecidos e } \\
\text { situações novas }\end{array}$} & Média & 1,80 & 1,95 \\
\hline & DP & 0,96 & 1,08 \\
\hline & Valor Mín. & 0 & 0 \\
\hline & Valor Máx. & 4,0 & 4,0 \\
\hline \multirow{4}{*}{$\begin{array}{l}\text { F5- Autocontrole da } \\
\text { Agressividade }\end{array}$} & Média & 2,83 & 3,06 \\
\hline & DP & 0,97 & 0,91 \\
\hline & Valor Mín. & 0 & 0 \\
\hline & Valor Máx. & 4,0 & 4,0 \\
\hline
\end{tabular}

Diante desse resultado, procedeu-se à comparação entre os itens que compõem o F5, a qual identificou que somente o item 38 "Você consegue 'levar na esportiva' as gozações de colegas de escola ou do trabalho a seu respeito" apresentou uma diferença significativa $\left(X^{2}=14,1 ; \mathrm{gl}=4 ; \mathrm{p}=0,007\right)$ entre os grupos, enquanto os dois outros itens que compõem esse fator, sendo eles o item "Quando um de seus familiares, por algum motivo te critica você reage de forma agressiva" e o item "Ao entrar em um ambiente onde estão várias pessoas desconhecidas, você cumprimenta-as" não apresentaram diferenças estatisticamente significativas.

\section{Discussão}

Ao analisar a literatura (Felicissimo et al., 2013) acerca da relação entre alcoolismo e habilidades sociais, percebe-se um aumento em intervenções que utilizam o Treinamento de Habilidades Sociais como tratamento principal ou coadjuvante para a dependência de álcool. No entanto, como encontrado na revisão realizada por Felicissimo et al. (2013), não existem evidências suficientes que comprovem a existência de um déficit no repertório de habilidades sociais em alcoolistas, o que serviu como ponto de partida para o presente estudo.

Assim, este estudo buscou investigar a existência de diferenças quanto ao repertório de habilidades sociais em alcoolistas e não alcoolistas. Os resultados encontrados não foram suficientes para ratificar essa associação, apesar de apresentar avanços comparados aos estudos já existentes nessa área.

O estudo de Aliane et al. (2006) aponta como uma importante limitação o tamanho amostral utilizado em seu estudo, constituído por 80 sujeitos, sendo 40 dependentes de álcool e 40 não dependentes. A amostra reduzida pode ter conduzido a um erro do Tipo II, que consiste em não encontrar uma diferença estatisticamente significativa em função do tamanho amostral, indicando a necessidade de realizar estudos com amostras maiores. Nesse sentido, o presente estudo utilizou uma amostra quase três vezes maior do que o estudo supracitado e, de fato, encontrou resultados diferentes.

Foi encontrada uma diferença significativa entre dependentes e não dependentes de álcool quanto ao F5 "Autocontrole da agressividade", que avalia especificamente a capacidade do participante em reagir a estimulações aversivas do interlocutor com razoável controle da raiva e da agressividade. Para indivíduos com um bom repertório de habilidades sociais nessa categoria, é possível expressar desagrado ou raiva de forma socialmente competente (Del Prette \& Del Prette, 2001).

Entretanto, a fim de melhor explorar esse achado, o presente estudo identificou que apenas um item representante dessa classe de habilidades sociais apresentava diferença significativa entre as amostras, limitando a mesma quanto à habilidade de lidar com humilhações e brincadeiras de conhecidos. Essa habilidade expressa uma característica importante para muitas demandas interpessoais, qual seja, a capacidade de lidar com conflitos de forma habilidosa (Del Prette \& Del Prette, 2001). Segundo Caballo (2010), faltariam 
aos alcoolistas principalmente as habilidades necessárias para lidar com situações de conflito, e, portanto, o consumo de álcool serviria como uma estratégia de enfrentamento a tais situações sociais ansiogênicas. Posteriormente, a frequência desse comportamento levaria a um aprendizado parcial e inadequado, reforçando a idéia da necessidade do consumo de álcool para o enfrentamento de situações cotidianas (Caballo, 2010). Sendo assim, existiria uma relação de influência múltipla, em que o álcool poderia estar sendo consumido como forma de enfrentamento a situações sociais ansiogênicas e, por sua vez, conduziria ao aprendizado inadequado de enfrentamento a essas situações, limitando o desenvolvimento de um repertório de habilidades sociais adequado.

Tais resultados estão em acordo com o estudo de Wagner, Silva, Zanettelo e Oliveira (2010) e Wagner e Oliveira (2009), em que se compara o repertório de habilidades sociais de dependentes e não dependentes de maconha com o mesmo perfil sociodemográfico e, igualmente ao presente estudo, encontraram diferenças significativas no F5 "Autocontrole da agressividade". Este resultado indica que dependentes de maconha, semelhante aos dependentes de álcool, possuem maior inabilidade para lidar com sentimentos e reações de agressividade, o que pode contribuir para o comportamento de buscar uma substância para enfrentar dificuldades sociais, além de prejudicar suas relações sociais ao reagir com baixo controle sob a raiva em situações de conflitos.

O trabalho de Scali e Ronzani (2007) reforça esse relacionamento, pois, ao investigar diferenças entre crenças relacionadas ao consumo de álcool entre dependentes e não dependentes dessa substância, encontraram que alcoolistas acreditam que o álcool diminui e/ou atua como uma fuga para emoções negativas e, consequentemente, fazem uso dessa substância quando se deparam com tais situações. Ademais, Cunha et al. (2007) concluíram que a ingestão etílica é vista como um facilitador do desempenho social, proporcionando maior ousadia, coragem, confiança, entre outras características, aos dependentes de álcool.

Por último, Cunha et al. (2012) realizaram um estudo exploratório quanto à relação entre o beber problemático e o déficit no repertório de habilidades sociais entre jovens universitários. Os autores encontraram uma prevalência de $10,6 \%$ dos universitários que bebiam de forma problemática com déficits no F5 comparados à amostra universitária normativa do IHS, reforçando a associação entre pior desempenho nesse fator e consumo de álcool.

Por outro lado, o resultado deste estudo difere do apresentado por Cunha et al. (2007), em que os autores encontraram déficits no repertório de habilidades sociais de dependentes de álcool nos fatores de "autoafirmação de sentimento positivo" e "conversação e desenvoltura social". Contudo, o grupo que serviu de comparação com a amostra de alcoolistas foi composto por jovens adultos e universitários. É possível hipotetizar que, ao comparar uma amostra de dependentes de álcool com uma amostra de jovens universitários, estes possuam uma desenvoltura social superior oriunda do processo de escolarização e desenvolvimento exigido pela academia e que portanto, os alcoolistas apresentariam um desempenho inferior comparados à eles.
No entanto, apesar dos resultados apresentados, ainda é preciso desenvolver mais estudos que fundamentem a relação entre déficit de habilidades sociais e alcoolismo, visto que os estudos desenvolvidos até o momento apresentam resultados diversificados. É preciso investir em estudos longitudinais que aprofundem os achados a fim de investigar se o déficit na classe de autocontrole da agressividade encontrada na presente amostra antecede aos comportamentos agressivos de dependentes de álcool, se a relação é contrária, ou se é uma relação de múltipla influência.

Além disso, o presente estudo apresenta algumas limitações que precisam ser consideradas ao analisar os resultados. A amostra estudada foi selecionada pelo critério de conveniência em ambos os grupos, o que limita a generalização dos resultados, visto que não é possível inferir se os dados representam o perfil de todos os dependentes de álcool que buscam tratamento para sua condição de saúde. Entretanto, vale destacar que outros estudos conduzidos em contextos diversos encontraram o mesmo perfil, dando suporte para a representatividade dos resultados encontrados. Ainda, a avaliação do repertório de habilidades sociais se deu através de um instrumento de autorrelato, que possui limitações inerentes ao próprio instrumento, visto que avalia a percepção do desempenho do repertório de habilidades sociais do próprio indivíduo, não sendo possível assegurar que o mesmo se comporte da forma como foi relatado.

Apesar das limitações apresentadas, este estudo representa avanços para pesquisas na área, uma vez que utiliza um tamanho amostral superior aos estudos já existentes e utiliza como amostra comparativa indivíduos com o mesmo perfil sociodemográfico, aumentando a segurança na comparabilidade entre os grupos, já que variáveis que poderiam influenciar o desenvolvimento do repertório de habilidades sociais foram controladas. Dessa forma, os resultados apresentados pelo atual estudo são inovadores e apontam para novas perspectivas de atuação nessa temática.

\section{Conclusão}

Os resultados não confirmaram a hipótese inicial de que dependentes de álcool possuem um menor repertório de habilidades sociais comparados a seus pares. No entanto, foram encontradas diferenças no fator representante da habilidade de autocontrole da agressividade, demonstrando um pior desempenho dessa habilidade em alcoolistas e indicando que as diferenças existentes entre os grupos são restritas a um único fator. Dessa forma, sugere-se que a avaliação do repertório de habilidades sociais em pacientes alcoolistas deva ser considerada em seu tratamento e que o desenvolvimento de um treinamento das habilidades deficitárias possa ser associado ao plano terapêutico, quando tais déficits forem encontrados.

\section{Referências}

Aliane, P. P., Lourenço, L. M., \& Ronzani, T. M. (2006). Estudo comparativo das habilidades sociais de dependentes e não dependentes de álcool. Psicologia em Estudo, 11(1), 83-88. 
Amorim, P. (2000). Mini International Neuropsychiatric Interview (MINI): Validação de entrevista breve para diagnóstico de transtornos mentais. Revista Brasileira de Psiquiatria, 22(3), 106-115.

Caballo, V. E. (2010). Manual de avaliação e treinamento das Habilidades Sociais. São Paulo: Santos.

Costa, J. S. D., Silveira, M. F., Gazalle, F. K., Oliveira, S. S., Hallal, P. C., Menezes, A. M. B., ... Macedo, S. (2004). Consumo abusivo de álcool e fatores associados: Estudo de base populacional. Revista de Saúde Pública, 38(2), 284-291.

Cunha, S. M., Peuker, A. C., \& Bizarro, L. (2012). Consumo de álcool de risco e repertório de habilidades sociais entre universitários. Psico, 43(3), 289-297.

Cunha, S. M., Carvalho, J. C. N., Kolling, N.M., Silva, C. R., \& Kristensen, C. H. (2007). Habilidades sociais em alcoolistas: Um estudo exploratório. Revista Brasileira de Terapias Cognitivas, 3(1), 28-41.

Del Prette, A., \& Del Prette, Z. A. P. (2001). Inventário de Habilidades Sociais (IHS-Del-Prette): Manual de aplicação, apuração e interpretação. Casa do Psicólogo.

Duarte, P. C. A. V., Stempliuk, V. A., \& Barroso, L. P. (2009). Relatório Brasileiro sobre Drogas. Brasilia: Secretaria Nacional de Politicas sobre Drogas.

Felicissimo, F. B., Casela, A. L. M., \& Ronzani, T. M. (2013). Habilidades Sociais e alcoolismo: Uma revisão da literatura. Psicologia em Estudo, 18(1), 137-145.
Gigliottia, A., \& Bessa, M. A. (2004). Síndrome de Dependência do Álcool: Critérios diagnósticos. Revista Brasileira de Psiquiatria, 26(1), 11-13.

Kadden, R. M., \& Cooney, N. L. (2005). Treating Alcohol Problems. In G.A. Marlatt \& D.M. Donovan (Eds.), Relapse Prevention (pp. 65-91). Nova Yorque: The Guilford Press.

Laranjeira, R., Pinsky, I., Zaleski, M., \& Caetano, R. (2007). I Levantamento Nacional sobre os padrões de consumo de álcool na população brasileira. Brasília: Secretaria Nacional Antidrogas (SENAD).

Monteiro, C. F. S., Albuquerque, I. E. M., Fé, L. C. M., Silva, M. G., Moreira, M. A. C., \& Passamani, M. C. (2011). Perfil sociodemográfico e adesão ao tratamento de dependentes de álcool em Caps-ad do Piauí. Revista de Enfermagem Escola de Enfermagem Anna Nery, 15(1), 90-95.

Scali, D. F., \& Ronzani, T. M. (2007). Estudo das expectativas e crenças pessoais acerca do uso de álcool. Revista Eletrônica Saúde Mental Álcool e Drogas, 3(1), 1 - 14.

Wagner, M. F., Silva, J. G., Zanettelo, L. B., \& Oliveira, M. S. (2010). O uso da maconha associado ao déficit de habilidades sociais em adolescentes. Revista Eletrônica Saúde Mental Álcool e Drogas, 6(2), 255 - 273.

Wagner, M. F., \& Oliveira, M. S. (2009). Estudo das habilidades sociais em adolescentes usuários de maconha. Psicologia em Estudo, 14(1), $101-110$.

WHO. (2014). Global Status Report on Alcohol and health 2014. Geneva: Department of Mental Health and Substance Abuse.

Recebido em 13.01.2015

Primeira decisão editorial em 09.02.2016

Versão final em 12.02.2016

Aceito em 12.02.2016 